\title{
Effects of a mixed exercise program on physical and cognitive condition: differences between older adults with and without dementia
}

\author{
Vazini Taher A. ${ }^{1 \mathrm{ABCD}}$, Skrypchenko I.T. ${ }^{2 \mathrm{ACDE}}$, Shuba V.V. ${ }^{3 \mathrm{ACDE}}$ \\ ${ }^{1}$ Faculty of Physical Education, Razi University of Kermanshah, Iran \\ ${ }^{2}$ Water Sport Department, Prydniprovsk State Academy of Physical Culture and Sports, Ukraine \\ ${ }^{3}$ Pedagogy and Psychology Department, Prydniprovsk State Academy of Physical Culture and Sports, Ukraine
}

Authors' Contribution: A - Study design; B - Data collection; C - Statistical analysis; D - Manuscript Preparation; E - Funds Collection.

\begin{abstract}
Purpose: $\quad$ The purpose of this study was to quantify the effects of a training protocol on physical and cognitive condition in a sample of individuals dwelling in a health care center for older persons.

Material: $\quad$ A 12 weeks mixed motor cognitive training with 8 weeks follow up was performed by 20 individuals with dementia and 20 individuals with intact cognitive state (mean age $=76 \pm 7$ ). Main outcome measures included body mass index, performance-oriented balance, frailty, performance in daily activities and mental abilities.

Results: At post intervention tests performance-oriented balance improved in both group. There was also a reduction in BMI in the normal group. These changes lasted after 8 weeks of no training. Analysis over time and between groups after the intervention showed a significant change in FR test $(p=0.001)$. Performance-oriented balance was improved in both groups regardless to initial cognitive state and somehow it was affected more in cognitively intact individuals.

Conclusions: Although these findings support the dominant effect of our training protocol on physical aspects, the benefits of training on cognition is not decisive.

Keywords: combined exercise, performance-oriented balance, cognition, elderly.
\end{abstract}

\section{Introduction}

The prevalence of dementia rises as the person get older than 65 years and it impacts his/her ability to maintain physical and mental function [1]. Exercise helps to reduce the risk of developing secondary conditions that come with functional decline. Combined exercises that focus on functional fitness factors, such as balance, is associated with significant improvements in the levels of independence in older adults [2, 3].

Poor stability in older adults is consistent with slower walking, increased stride variability, higher fall rate, and poorer performance on complex mobility tasks [4]. There are also many debates about the role of stability in falling and gait control. Jorgensen and colleagues found that balance was not a risk factor for falling in elderly. On the other hand, Lamb, Bartlett, Ashley and Bird (2002) and Hyndman, Ashburn and Stack (2002) showed that balance was a risk factor $[5,6]$. Nonetheless, the query of balance as a risk factor is not fully answered yet. Also, the evidence is not conclusive as to whether balance or mobility are enhanced by mixed exercises when the sample is individuals with dementia and intact cognitive status [7].

The mixed exercise programs combine a cognitive with a physical exercise, e.g. postural control exercises together with cognitive tasks or performing dualtasking exercises. The integrated motor and cognitive components are key features of these programs. This involves a greater interest for dual- or multiple-task

(c) Vazini Taher A., Skrypchenko I.T., Shuba V.V., 2019 practice than other attention-demanding exercise forms (e.g., Tai Chi). Performing dual task needs the persons to divide their attention, which can interfere with gait and balance control [8]. It is already demonstrated that in elderly people with dementia, cognitive status would have a relationship with functional performance (e.g. gait and balance). This association would be particularly evident during walking under dual-task conditions [9].

Although there is rewarding evidence on the benefits of exercise for the health and functioning of older adults with cognitive disorders [10], the clinical evidence lacks supportive recommendations for exercise and testing in older people with dementia [11]. Studies on exercise and Alzheimer's disease or related dementia often include small sample sizes and report inconsistent positive findings [10]. For this reason, more studies with well described methodology and training programs are needed for evidence-based outcomes of interventions aiming to improve health and performance in elderly population. The findings of present study should be useful in clarifying the effects of exercise training for cognitively impaired older adults, especially persons with dementia.

Regarding above mentioned matters, we hypothesize that motor cognitive exercise using dual task condition could be an efficient method to enhance physical condition and cognitive status in older people with cognitive impairments, like dementia. The purpose of this study was to evaluate the effects of a mixed exercise program on physical and cognitive condition between older adults with and without dementia. 


\section{Material and methods}

Research Design

A randomized controlled trial with a 2 month follow up was performed, with data collection from spring to summer 2017.

\section{Participants}

Forty elderly men and women aged 65 years old and higher were recruited from a health care center located in the city of Hamedan, Iran. Our study was designed to include 20 subjects in each cognitive group and in each gender group. The researcher evaluated the subject's appropriateness for inclusion in the study by administering a medical history questionnaire. Four inclusion criteria were used: (1) not dependent on the assistance of another person or the assistance of a support device (e.g. cane, walker, crutch) for walking, (2) 65 years of age or older, (3) no history of dizziness. Exclusion criteria were the diagnosis of a stroke, Parkinson's disease, or other neurological disorder, the presence of a severe cognitive impairment, symptomatic cardiovascular disease, and peripheral neuropathy of the lower extremities. The informed consent form was filled by the participants. Ethical approval for research involving human subjects sought and received via the Hamedan Welfare Organization ethics review procedure.

Procedures

Data were collected within a 45 minute test session for each subject in January and April of 2015 for pre and post intervention assessments, and June 2015 for follow up measurements. Testing was conducted in a special place, which were reserved uniquely for data collection on test days. Informed consent was acquired when the subject came into the session. Demographic data were collected in order to describe the study sample (age, height, weight, medical diagnoses, resting blood pressure, and heart rate). Subjects also answered several questions concerning daily activities in order to describe the activity level of the participants, as well as MMSE as a measure of cognitive status. The physical condition tests were administered to each subject in the same order: 1) BBS and 2) FR. All examiners were trained in the standardized instructions for administering the tests.

The training protocol consisted of physical exercises with low to high cognitive load and covered two types of challenging demands; 1) motor demands such as changing center of gravity, alternative gait, and moving limb in full range of motion, 2) cognitive demands like attention, quick response to visual stimulus, decision making, and response inhibition. Intensity and duration of the program were chosen based on the guidelines published by the American College of Sports Medicine [12]. Training sessions were conducted in groups of four or five participants to encourage exercise class participation and to form group cohesion [13]. A training session lasted on average 45 minutes and consisted of 6 cognitive-motor exercises in 2-3 sets of 5-10 repetitions. Training intensity was controlled by a perceived exertion rate. To maintain the intensity of the stimulus during the training period, the number of repetitions and the cognitive load were progressively increased as tolerated by the participants. As such, the training program was designed to include three levels (A, B, and $\mathrm{C}$ ) in which the cognitive and physical load was progressively increased from level A (lowest load) to Level C (highest load). All participants started the training at level A and only after mastering this level progressed to the next level.

The following tests were performed in a week before and after the 12 weeks training period in a suitable room designed for this purpose at the health care center.

\section{Berg Balance Scale}

The Berg Balance Scale (BBS) with 14 items (scored on a scale of 0 to 4 ) is developed to measure performanceoriented balance in elderly individuals. For a participant who is unable to do the task, a 0 score is recorded and a participant who is able to complete the task scores 4 . The overall score of BBS is 56 . The items include simple mobility tasks like standing unsupported and more difficult tasks like single-leg stance. Studies of various elderly populations have shown high intra-rater and interrater reliability ratio of variability. Content validity of the BBS is already established in geriatric settings. Criterion-related validity has been supported by moderate to high correlations between BBS scores and other functional measurements in a variety of older adults with disability [14].

\section{FR Functional Reach}

Functional reach (FR) is a new clinically accessible measure of balance and it equals the furthest distance in front of the body that a person, standing in a static position with arms fully prolonged, can touch without falling. The FR test, as originally devised by Duncan et al, is a measure of frailty in addition to an assessment of balance, flexibility, and fall risk. Norms for this test are: reach $<$ 6 inches $=$ high risk of falls/frailty; reach $>6$ inches and $<10$ inches $=$ moderate risk for falls/frailty; reach $>10$ inches $=$ low risk for falls/frailty [15].

Barthel Daily activities Index

The Barthel Index is an ordinal scale used to measure performance in activities of daily living (ADL). The performance items of ADL are rated with a given number of points assigned to each level or ranking. It uses ten variables describing ADL and mobility. The higher scores in ADL are related with more possibility of being able to live at home with a degree of independence following discharge from hospital. It is a simple to administer tool for assessing self-care and mobility activities of daily living. It is widely used in geriatric assessment settings. Reliability, validity and overall utility of ADL are range from good to excellent. Information is gained from observation, self-report or informant report [16].

\section{MMSE Mini Mental State Evaluation}

The Mini Mental State Examination (MMSE) is the most commonly used test for complaints of problems with memory or other mental abilities. The maximum MMSE score is 30 points. Scores ranged from 20 to 24 suggest mild dementia, scores ranged from 13 to 20 suggest moderate dementia, and scores less than 12 indicates severe dementia. This tool is used extensively in clinical 
and research settings to measure cognitive impairment. MMSE can be used by clinicians for initial diagnosis of dementia and to help assess the progression and severity of dementia and mild cognitive impairment. It is also used to follow the course of cognitive changes in an individual over time [17].

\section{Statistical Analysis}

Data were analyzed using SPSS software package (version 22.0). Descriptive statistics and frequencies were determined for demographic variables. Differences between groups were analyzed by calculating the difference between baseline and after three months. The calculated difference was used when comparing the difference between groups at three months using the Mann-Whitney U test. Normally distributed quantitative data were investigated with two-factor repeated-measures ANOVA, as a mixed design, main effect of factor 1 (time) and factor 2 (group) and interaction effects of factors 1 and 2 . The significance level was set at $\mathrm{P} \leq 0.05$ for all analysis.

\section{Results}

\section{Baseline scores}

The mean age of the participants who entered the trial was 75.65 years (range, 65-90). The values for basic characteristics, cognition and different functions for the all tests of subjects are shown in table 1. At baseline, there were no significant differences between groups regarding demographic characteristics. However, the mean scores of MMSE was significantly higher in normal group. Moreover, an analysis of separate questions from Barthel daily activities index showed significant differences between the two groups in two of the 10 questions, namely transfers $(\mathrm{p}=0.034)$ and mobility $(\mathrm{p}=0.38)$, but there was no significant difference in other items.

\section{Exercise effects}

There were two dropouts in normal group (1 man and 1 woman) due to discharging from center, though, all subjects in dementia group were persistent in whole period of training. Thus, adherence rate to the training sessions was more than 80 percent (mean $89 \%$ ).

The post intervention assessments showed that both groups experienced significant improvements after training period. The BMI of the dementia group was unchanged after training, but it had significantly decreased by $14 \%$ in the normal group $(\mathrm{p}=0.04)$. On the other hand, cognitive state was significantly improved in the dementia group, whereas there was no change in the normal group $(p=0.01)$. The effects of exercise on outcome measures are shown in figure 1.

Examinations after 12 weeks showed that, the distance covered in FR test was unchanged in the dementia group, whereas it was significantly increased by $21 \%$ in the normal group $(\mathrm{p}=0.02)$, demonstrating an increase of flexibility. The normal group scored higher in the BBS $(p=0.001)$. There was no change in the dementia group performance of this test.

There were no significant differences between the groups after three months for barthel index. However, there was a significant decrease in the BMI in favor of normal group. The results of tests after 8 weeks of no training showed that both groups maintained all the changes occurred in training period. Analysis over time and between groups after the intervention revealed no difference in BBS test, but a significant change in FR test was shown $(\mathrm{p}=0.001)$.

\section{Discussion}

Little comparative studies have been conducted on the impact mixed exercise has on physical and cognitive outcomes in persons with and without dementia.

The purpose of this study was to examine the effects of a combined motor-cognitive training protocol on cognition, performance - oriented balance and health in older people with/without dementia. We expected a lower cognitive state in the dementia group compared to the group without dementia. In addition, we hypothesized that people in the dementia group would have more difficulty performing performance - oriented balance, resulting in a more pronounced motor-cognitive exercise effect on balance tests. Nonetheless, two groups significantly differed in the results of FR test after training period (in favor of normal group).

The current study demonstrated that in older people living in care center, a 12 weeks combined exercise program improved cognitive status, physical condition

Table 1. Baseline characteristics of participants

\begin{tabular}{llll}
\hline Factors & Dementia (20) & Normal (20) & Overall (40) \\
\hline Age & $78.60(7.79)$ & $72.70(5.85)$ & $75.65(7.42)$ \\
Height & $160.80(6.21)$ & $160.25(7.76)$ & $160.53(6.94)$ \\
Weight & $66(6.31)$ & $61.55(8.72)$ & $63.78(7.84)$ \\
BMI & $24.53(2.14)$ & $23.88(1.90)$ & 24.20 \\
Sex (\% men) & 50 & 50 & 50 \\
MMSE & $16.44(2.18)$ & $25.57(2.15)$ & 21 \\
Education level (\% only primary school) & 81 & 76 & 78 \\
Medication (\% $\geq 2$ per day) & 36 & 29 & 32 \\
BBS & $33.65(10.44)$ & $37.60(7.54)$ & $35.63(9.21)$ \\
FR & $21.10(7.98)$ & $25.15(5.62)$ & 23.12 \\
Barthel index & $79.45(17.30)$ & $80.40(18.08)$ & $79.93(17.47)$ \\
\hline
\end{tabular}




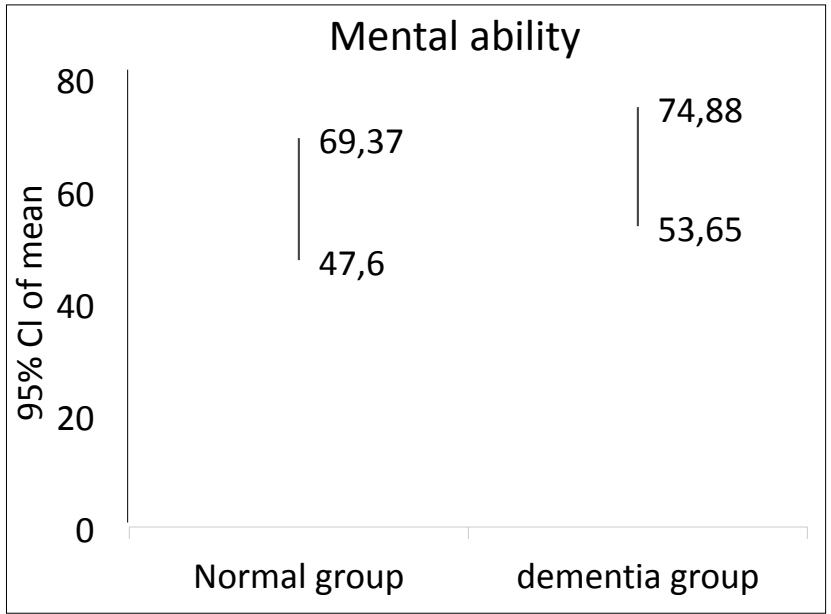

a)

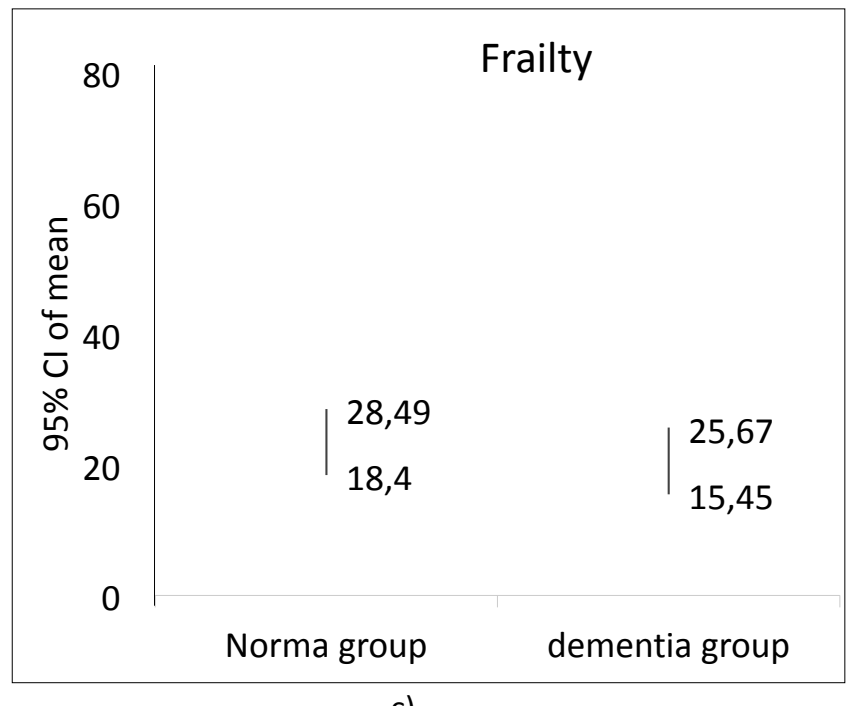

c)

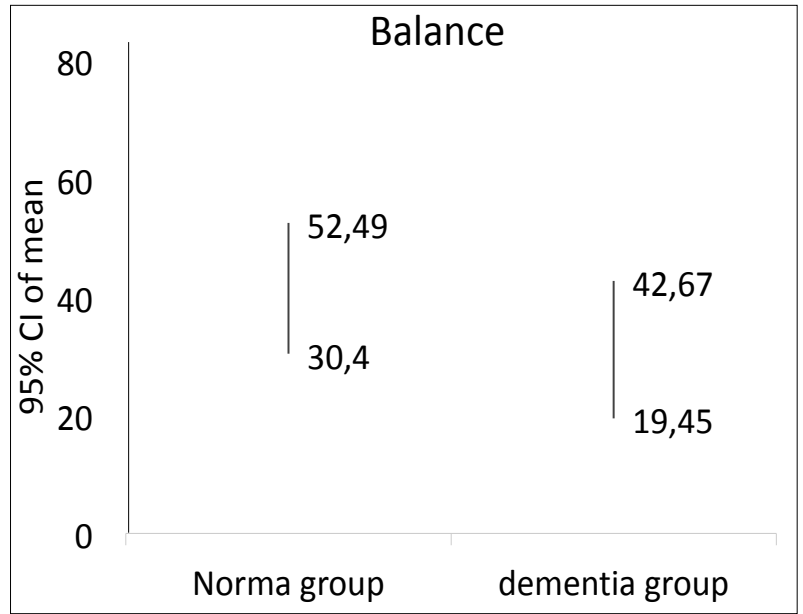

b)

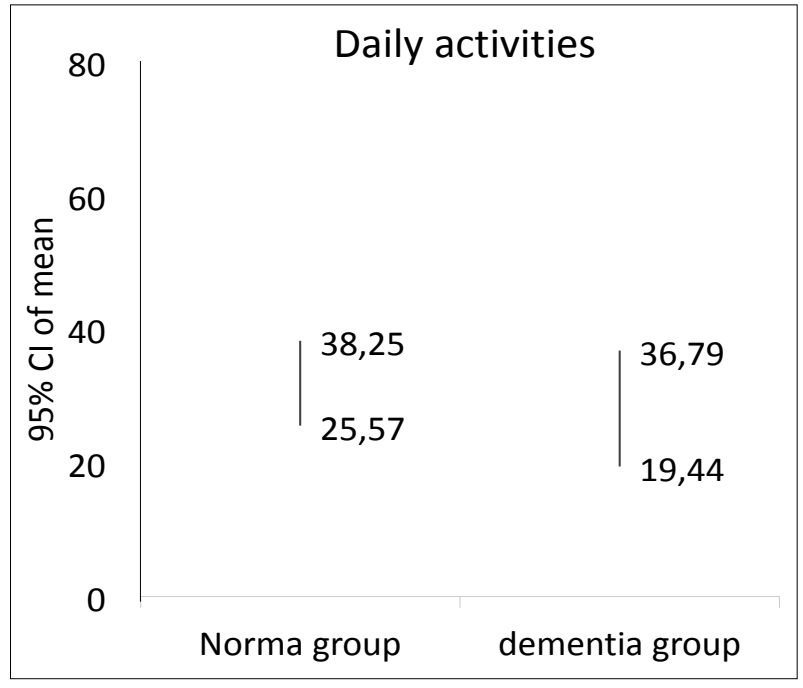

d)

Figure 1. The effects of exercise on outcome measures. The means and $95 \%$ confidence intervals (Cls) for two groups on (a) mental ability, (b) performance-oriented balance, (c) frailty (FR test) and (d) performance in daily activities.

and health both in individuals with dementia and intact cognitive state. However, dementia group experienced more changes in cognition, while the normal group had the most evident effects in health status and performance in FR test. Therefore, in contrast to what we assumed at first, performance - oriented balance was improved in both groups regardless to initial cognitive state and somehow it was affected more in cognitively intact individuals. Although these findings support the dominant effect of our training protocol on physical aspects, the benefits of training on cognition is not decisive.

The present study aimed to extend the prior work in some ways. First, previous studies explored physical condition with merely a single test. In contrast, we implemented a battery of tests, including two physical condition tests (i.e. BBS and FR). These tests were used because falling in older ages is highly prevalent. Also, the effects of motor-cognitive training on balance/postural control is rarely evaluated. Nonetheless, the control of posture and balance are critical components in fall prevention. We also administered two different health tests (BMI and Barthel activity index) because execution of a balance test could be limited partially due to physical conditions. Second, most of previous works concentrated on a population of elderly people without dementia or other neurological disorders. Here, we recruited two groups of elderly persons with/without dementia living in a health care center. Last but not least, as cognition is linked with performance - oriented balance, this study assessed cognitive status prior and after training period. We should also remind that all measures were administered two months after last session of training which indicated that the results were consistent. A major strength of our study is that we examined both static and dynamic balance.

Shigematsu et al., administered Physical tests of balance, leg strength and coordination, which showed that Functional fitness of lower extremities improved more in training group than in control group [18]. Shigematsu et al., in another research used different measures of postural control and showed a significant within-group improvement in one leg balance [19].

Only two studies examined performance - oriented 
balance as an outcome for motor-cognitive training in older people. Silsupadol et al., found that BBS, DGI and $\mathrm{ABC}$ scores increased and Time to complete TUG decreased under Dual-task balance training [20]. Lamb et al., used a measure of walking (i.e., gait speed) along with specific one-item functional tests [5], whereas Hyndman et al., implemented just a functional measure (Rivermead Mobility Index) [6]. These researchers suggest that comprehensive assessments with focus on the physical, cognitive/ psychological, and environmental factors are important.

Our results fit in with the results of the group of Shigematsu and colleagues but are partially in contrast to the results reported by the groups of Silsupadol and colleagues. They found that Subject who received DTtraining, showed improvements on other dual tasks that were not directly trained. Future research should evaluate if a motor-cognitive training protocol can improve performance - oriented balance in older people with dementia.

\section{Practical application}

This study provides additional insight in the interaction of aging with cognitive status and postural control in elderly people living in a care center: motorcognitive training improved physical condition. This finding suggests that an exercise program combined with cognitive training is helpful to reduce the degenerative effects of dementia on older persons' cognition. In future research, the pathophysiology and further clinical implications should be investigated.

\section{Conflict of interests}

The authors declare that there is no conflict of interests.

\section{References}

1. Millan-Calenti JC, Tubío J, Pita-Fernández S, GonzálezAbraldes I, Lorenzo T, Fernandez-Arruty T, Maseda A. Prevalence of functional disability in activities of daily living (ADL), instrumental activities of daily living (IADL) and associated factors, as predictors of morbidity and mortality. Archives of gerontology and geriatrics. 2010;50(3):306- 10. https://doi.org/10.1016/j.archger.2009.04.017

2. Cadore EL, Rodríguez-Mañas L, Sinclair A, Izquierdo M. Effects of different exercise interventions on risk of falls, gait ability, and balance in physically frail older adults: a systematic review. Rejuvenation research. 2013;16(2):105- 14. https://doi.org/10.1089/rej.2012.1397

3. Paquette MR, Li Y, Hoekstra J, Bravo J. An 8-week reactive balance training program in older healthy adults: A preliminary investigation. Journal of Sport and Health Science. 2015;4(3):263- 9. https://doi.org/10.1016/j.jshs.2014.06.004

4. Ambrose AF, Paul G, Hausdorff JM. Risk factors for falls among older adults: a review of the literature. Maturitas. 2013;75(1):51- 61 . https://doi.org/10.1016/j.maturitas.2013.02.009

5. Lamb SE, Bartlett HP, Ashley A, Bird W. Can lay-led walking programmes increase physical activity in middle aged adults? A randomised controlled trial. Journal of Epidemiology \& Community Health. 2002;56(4):246- 52. https://doi.org/10.1136/jech.56.4.246

6. Hyndman D, Ashburn A, Stack E. Fall events among people with stroke living in the community: circumstances of falls and characteristics of fallers. Archives of physical medicine and rehabilitation. 2002;83(2):165- 70. https://doi.org/10.1053/apmr.2002.28030

7. Chang YK, Nien YH, Chen AG, Yan J. Tai Ji Quan, the brain, and cognition in older adults. Journal of Sport and Health Science. 2014;3(1):36- 42. https://doi.org/10.1016/j.jshs.2013.09.003

8. Woollacott M, Shumway-Cook A. Attention and the control of posture and gait: a review of an emerging area of research. Gait \& posture. 2002;16(1):1- 4. https://doi.org/10.1016/S0966-6362(01)00156-4

9. Scherder E, Eggermont L, Swaab D, van Heuvelen M, Kamsma Y, de Greef M, van Wijck R, Mulder T. Gait in ageing and associated dementias; its relationship with cognition. Neuroscience \& Biobehavioral Reviews. 2007;31(4):485- 97. https://doi.org/10.1016/j.neubiorev.2006.11.007
10.Heyn P, Abreu BC, Ottenbacher KJ. The effects of exercise training on elderly persons with cognitive impairment and dementia: A meta-analysis1. Archives of physical medicine and rehabilitation. 2004;85(10):1694- 704. https://doi.org/10.1016/j.apmr.2004.03.019

11.Hauer K, Schwenk M, Zieschang T, Essig M, Becker C, Oster P. Physical training improves motor performance in people with dementia: a randomized controlled trial. Journal of the American Geriatrics Society. 2012;60(1):8- 15. https://doi.org/10.1111/j.1532-5415.2011.03778.x

12.Pate RR, PrattM, Blair SN, Haskell WL, Macera CA, Bouchard C, Buchner D, Ettinger W, Heath GW, King AC, Kriska A. Physical activity and public health: a recommendation from the Centers for Disease Control and Prevention and the American College of Sports Medicine. Jama. 1995;273(5):402- 7. https://doi.org/10.1001/jama.1995.03520290054029

13. Midtgaard J, Rorth M, Stelter R, Adamsen L. The group matters: an explorative study of group cohesion and quality of life in cancer patients participating in physical exercise intervention during treatment. European Journal of Cancer Care. 2006;15(1):25- 33. https://doi.org/10.1111/j.1365-2354.2005.00616.x

14.Qutubuddin AA, Pegg PO, Cifu DX, Brown R, McNamee S, Carne W. Validating the Berg Balance Scale for patients with Parkinson's disease: a key to rehabilitation evaluation. Archives of physical medicine and rehabilitation. 2005;86(4):789- 92. https://doi.org/10.1016/j.apmr.2004.11.005

15.Sinoff G, Ore L. The Barthel Activities of Daily Living Index: self-reporting versus actual performance in the old-old ( $\geq 75$ years). Journal of the American Geriatrics Society. 1997;45(7):832- 6. https://doi.org/10.1111/j.1532-5415.1997.tb01510.x

16. Mitchell AJ. A meta-analysis of the accuracy of the mini-mental state examination in the detection of dementia and mild cognitive impairment. Journal of psychiatric research. 2009;43(4):411- 31. https://doi.org/10.1016/j.jpsychires.2008.04.014

17.Wernick-Robinson M, Krebs DE, Giorgetti MM. Functional reach: does it really measure dynamic balance?. Archives of physical medicine and rehabilitation. 1999;80(3):262-9. https://doi.org/10.1016/S0003-9993(99)90136-3

18.Shigematsu R, Okura T, Nakagaichi M, Tanaka K, Sakai T, Kitazumi S, Rantanen T. Square-stepping exercise and fall risk factors in older adults: a single-blind, randomized controlled trial. The Journals of Gerontology Series A: 
Biological Sciences and Medical Sciences. 2008;63(1):76-82. https://doi.org/10.1093/gerona/63.1.76

19.Shigematsu R, Okura T, Sakai T, Rantanen T. Square-stepping exercise versus strengthand balance training forfallrisk factors. Aging clinical and experimental research. 2008;20(1):19- 24. https://doi.org/10.1007/BF03324743
20.Silsupadol P, Siu KC, Shumway-Cook A, Woollacott MH. Training of balance under single-and dual-task conditions in older adults with balance impairment. Physical therapy. 2006;86(2):269-81.

\section{Information about the authors:}

Vazini Taher A.; http://orcid.org/0000-0001-7780-7054; vazinitaher@gmail.com; Faculty of Physical Education, Razi University of Kermanshah; Kermanshah, Iran.

Skrypchenko I.T.; (Corresponding author); http://orcid.org/0000-0001-5895-3099; sit71@ukr.net; Water Sport Department, Prydniprovsk State Academy of Physical Culture and Sports; st. Victory Embankment, 10, Dnipro, 49094,Ukraine.

Shuba V.V.; http://orcid.org/0000-0001-5042-3106; Victoria-shuba79@yandex.ru; Pedagogy and Psychology Department, Prydniprovsk State Academy of Physical Culture and Sports; st. Victory Embankment, 10, Dnipro, 49094,Ukraine.

Cite this article as:

Vazini Taher A, Skrypchenko IT, Shuba VV. Effects of a mixed exercise program on physical and cognitive condition:

Differences between older adults with and without dementia. Pedagogics, psychology, medical-biological problems of physical training and sports, 2019;23(2):96-101.

https://doi.org/10.15561/18189172.2019.0207

The electronic version of this article is the complete one and can be found online at: https://www.sportpedagogy.org.ua/index.php/PPS/issue/archive

This is an Open Access article distributed under the terms of the Creative Commons Attribution License, which permits unrestricted use, distribution, and reproduction in any medium, provided the original work is properly cited (http://creativecommons.org/licenses/by/4.0/deed.en).

Received: 30.12 .2018

Accepted: 06.02.2019; Published: 30.04.2019 